Case Report

\title{
SINGLE COMPLETE DENTURE IN MANDIBULAR ARCH OPPOSING NATURAL DENTITION - A CASE REPORT
}

\author{
Laxman Singh Kaira ${ }^{1} \&$ Richa Singh ${ }^{2}$ \\ ${ }^{1}$ Assistant Professor, ${ }^{2}$ Intern, Department of Dentistry, Institute of Dental Sciences, Bareilly \\ Veer Chandra Singh Garhwali Government Research Institute and Medical Sciences \\ Srikot, Srinagar Garhwal, Uttrakhand. \\ Correspondence: \\ Laxman Singh Kaira, \\ Faculty Residence, Institute of Dental Sciences, Srikot, Srinagar Garhwal,Uttrakhand, \\ Mobile : +91 96341 03245; E-mail : luckysinghkaira111@gmail.com
}

\begin{abstract}
:
Several difficulties are encountered in providing a successful, single complete denture treatment. This case report deals with successful rehabilitation of completely resorbed mandibular ridge opposing a full complement of natural teeth prosthetically incorporating metal denture base in place of the conventional Poly Methyl Methacrylate material to combat the masticatory forces from natural dentition and improve the longevity of the prosthetic rehabilitation, at the same time improving the strength of the mandibular denture base.
\end{abstract}

Keywords : residual ridge resorption, metal denture base, single complete denture

\section{Introduction :}

The situation in which a patient has become entirely edentulous in one jaw while retaining either all or some of his natural teeth in the others is not uncommon. Neither is it uncommon to find that the successful complete denture for such a patient is often very difficult and on occasions virtually impossible ${ }^{1}$.

There are two reasons for this difficulty. The first is related to firmness and rigidity with which the natural teeth are retained in the bone and the magnitude of the force they can resist or deliver $\mathrm{n}$ without discomfort or displacement. This force has been recorded as high as $198 \mathrm{lb}$ on a single molar teeth. This is in sharp contrast with the force which a complete denture, resting simply on the delicate mucosa of the ridge, can resist or deliver. This force has been established as being a maximum static load of $26 \mathrm{lb}^{2}$.

The second reason is related to the occlusal form of the Access this article online Quick Response Code

arifing
remaining natural teeth, which will of necessity dictate the occlusal form of the denture. The natural teeth may be overerupted or tilted and their cusps high and sharp. As a result, occlusion and articulation will involve contacting of the inclined planes of the cusps in such a way that the denture will continually be thrust or dragged horizontally on the ridge ${ }^{2}$.

To overcome these problems, two things are necessary. First, full use must be made of every factor which favours success, and no minor error or imperfection which might perhaps have been tolerated in conventional complete denture construction should be accepted. Second, the forces to which the denture is subject must be reduced as much as possible by appropriate preparation or restoration of the remaining natural teeth so as to provide an acceptable occluding surface ${ }^{2}$.

\section{Clinical Report :}

A 43 year old male reported to the Department of Prosthetic Dentistry of Darshan dental college and Hospital, Udaipur, Rajasthan with a chief complaint of completely edentulous mandibular arch. Intraoral examination revealed highly resorbed mandibular ridge (fig 1) and opposing full complement of natural dentition. Mucosa was normal and the opposing teeth required minor alterations. Saliva was of medium consistency and patient was co-operative and philosophical according to House classification. Hence metal mandibular denture was planned for this patient. 


\section{Treatment Procedure :}

Impression of the upper natural teeth was made with a irreversible hydrocolloid impression material (Neocolloid, Zermack clinical, Italy) and an artificial stone diagnostic cast was poured. Preliminary impressions of the edentulous mandible was made with a viscous mixture of two varieties of softened impression compound (3 parts impression compound +7 parts greenstick compound) [McCord's Technique] The impressions were washed and poured with the dental plaster. The custom tray was prepared with auto-polymerising acrylic resin (DPI -RR cold cure, Dental Products of India, the Bombay Burmah Trading Corporation limited)) for making secondary impression. The seconday impression was made using green stick compound for border moulding ( DPI Pinnacle Tracing Sticks,Bombay, India) and medium body as impression material (Impregnum Penta Medium Body, Pearson Dental Supplies). Master cast was made with

Dental stone type III (Kalastone, Kalabhai Dental Private limited) and the mould of the same was made with reversible hydrocolloid ( agar agar) . A refractory cast was poured with ethyl silicate bonded investment material. On the refractory cast, the denture base pattern wax was adapted and the sprues were attached and invested( fig 2 \& fig 3$)$. The denture base was casted with cobalt chromium metal.

The jaw relations were recorded. Face Bow transfer and jaw relations were then verified and secured in a semiadjustable articulator for teeth arrangement. Teeth were arranged in centric occlusion so that the centric relation records could be verified. The anterior teeth were arranged provisionally for esthetics. The eccentric relation records were made in wax ant the condylar elements of the articulator was adjusted. Posterior teeth were rearranged to satisfy the requirements of balanced occlusion. Adjustments in the artificial teeth were incorporated in preference to making natural teeth. Though a perfect balanced occlusion is impossible to achieved in such cases with involvement of natural teeth, a maximum effort was made to get an occlusion which was as close to balanced occlusion.
A trial of waxed up mandibular denture was made (fig 4) followed by acrylization of the complete denture with heat polymerizing acrylic resin ( Trevalon, Dentsply, Gurgaon ,India) (fig 5 ). The interferences in the denture were eliminated and denture given to the patient. Post insertion instructions were given to the patient regarding its maintenance, nutrition and hygiene ( fig 6 \& fig 7).

\section{Discussion :}

One of the common clinical situations involving a single denture is that of a complete lower denture and upper natural teeth. When a complete denture is opposed by natural teeth, it will require some degree of contouring to provide a harmonious occlusion. The reasons for such alteration is mainly due to (1) Unfavourable inclination of the occlusal plane (2) malpositioned individual teeth which have assumed positions resulting excessively steep cuspal inclinations, and (3) to wide buccolingual width of the natural teeth ${ }^{3}$.

Balance in natural dentitions is mainly seen only in centric occlusion but not in the protrusive or lateral excursions. Protrusive and bilateral balance is not necessary for tooth retention as natural teeth resist stress from all directions individually and are thus stable. On the other hand, artificial dentures merely rest on the surface of the tissues with a film of saliva between the dentures and the mucosa. The teeth and the base functions as one unit and force exerted against any toothin an unfavourable direction dislodges the whole denture. Dentures remain stable only when forces exerted against the teeth are directed favourably to the base support. Horizontal thrust not only tends to dislodge the dentures but also tends to destroy the residual ridge ${ }^{4}$.

Mandibular denture bases may encounter tissue changes of the residual ridge followed by discomfort, occlusal problems and fracture of denture bases. There might be occlusal stress on the mandibular denture and the underlying edentulous tissue due to forces from teeth and musculature and opposing natural teeth, and the position of the maxillary teeth, which are improperly aligned may also avoid achievement of bilateral balance for stability and 


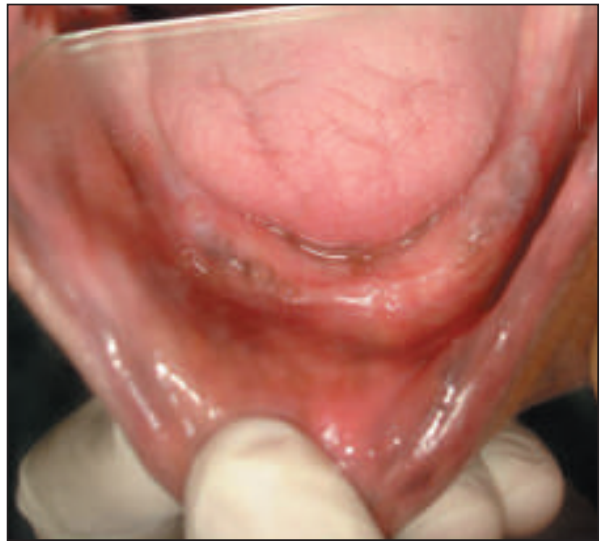

Figure 1 - Preoperative Photograph

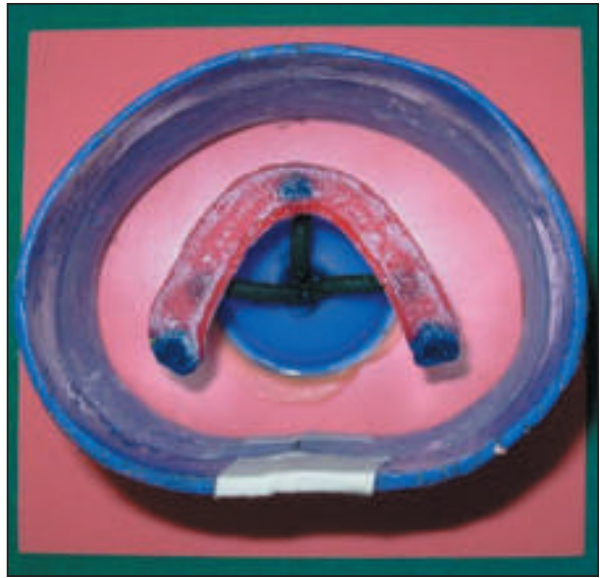

Figure 3 - Investing

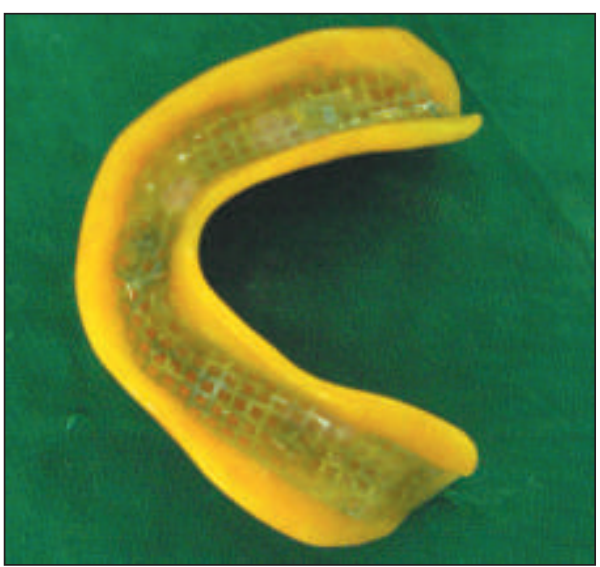

Fig 5 - Palmar View of Mandibular Denture Base

lead flexure of the denture bases ${ }^{5}$. The midline fracture in a denture is often a result of flexural fatigue. Though poly Methyl Metha Acrylate denture bases have good mechanical, biological and esthetic properties, the impact and fatigue strength of PMMA are not entirely satisfactory ,thus may fail when there is excessive parafunctional and / or functional forces ${ }^{6,7}$.

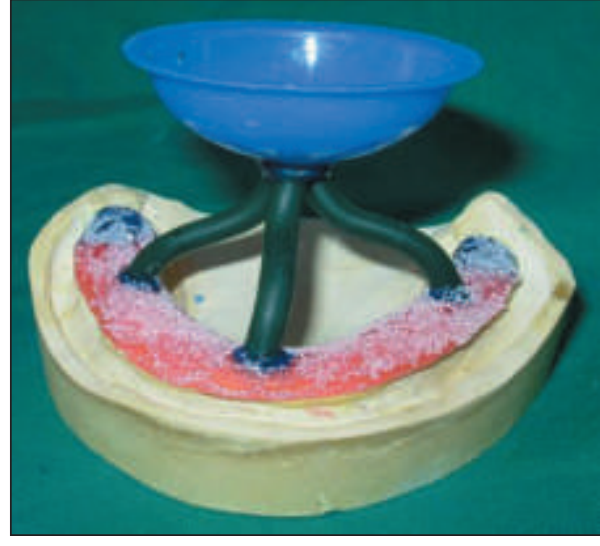

Figure 2 - Wax Pattern

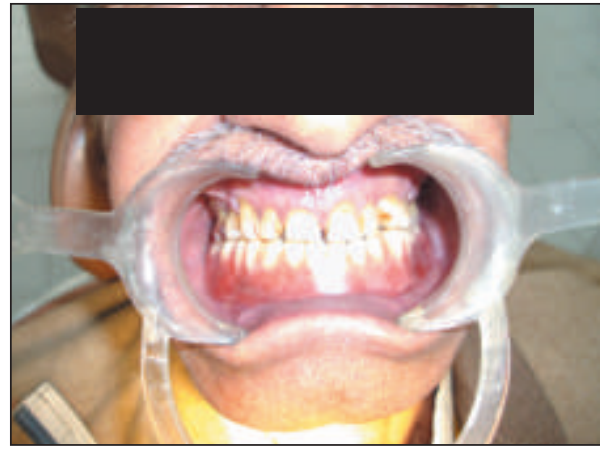

Fig 4 - Try In

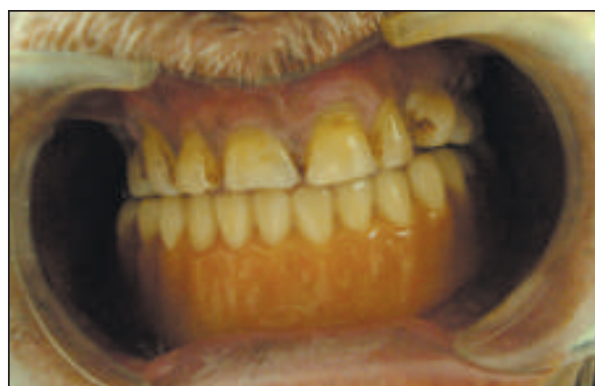

Fig 6 - Postoperative Photograph

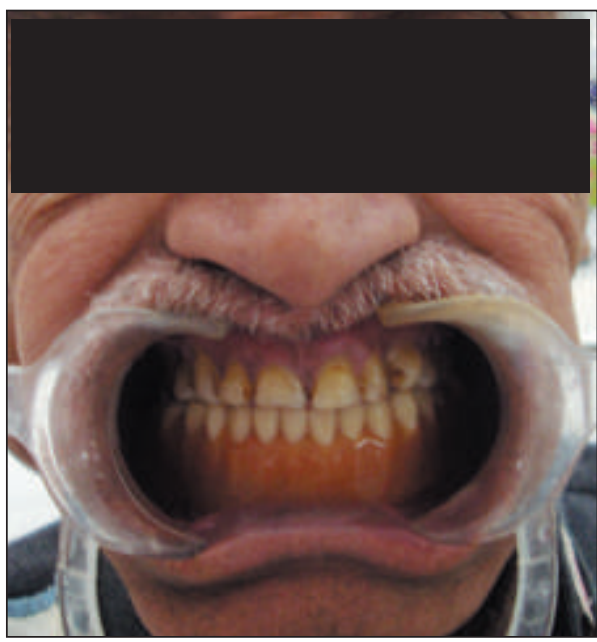

Fig 7 - Happy Patient 
Cobalt chromium bases in mandibular denture reduces functional deformation and thrust to the supporting tissues occurring in the anterior part of mandible ${ }^{8}$. Besides rigidity and fracture resistance these metal bases have several other advantages like excellent strength to volume ratio, good adaptation to the supporting tissues, enhanced plaque control, high thermal conductivity, very little dimensional changes in time through fluid absorption ${ }^{9}$

\section{Conclusion :}

This is the first case report that shows mandibular metal

References:

1. Sharry JJ. Complete Denture Prosthodontics. 3rd Ed. New York: McGraw-Hill; 1974; 310-311

2. Anderson, R. Storer . Immediate and Replacements Dentures, Blackwell Scientific Publications, Oxford, 1966; 4

3. Ellinger CW., Jack H. Rayson, Davis Henderson. Single complete denture. J Prosthet Dent , 1971;26(1):4-10

4. French FA. The problem of building satisfactory dentures. J Prosthet Dent, 1954; 4: 769-81.

5. Alex Koper. The maxillary complete denture opposing natural teeth : problems and some solutions. J Prosthet Dent1987; 57(6):704-7..

6. Ohkube C., Kurtz K .S., Suzuki Y., Hanatani S.,AbeM., Hosoi T. comparative study of maxillary complete dentures constructed of metal base and metal structure framework. Journal of oral rehabilitation, 2001;28: 1 49-56.

7. Schneider RL,Stokes JL,Laduke D. Design and fabrication technique for metal palates in maxillary complete dentures. J Dent Technol.2000; 17(7):8-11.

8. Schmizu H, Tsue F, Obukuro M , Kido H, Takahashi Y, OhmuroH. Fracture strength of metal base complete maxillary dentures with a newly designed metal framework .Int Chin J Dent.2005;5: 35-8.

9. Marcaubeanu C, Goguba L, Jivanescu A, Demjan E, Bratu D. Titanium complete denture base in a patient with heavy bruxism : a clinical report .Journal of Experimental Medical and Surgical Research. 2008; 3: 96-9. denture base in single complete denture. This combination i.e. mandibular metal base and opposing natural dentition provided great comfort to the patient as the metal denture base was strong to resist catastrophic failure and flexural fatigue if PMMA was to be used as denture base. The metal denture bases are good thermal conductors and less bulky. There would be no propagation of crack from the deep labial notch as well. 